\title{
ESTIMAÇÃO DE MAGNITUDE DA GRAVIDADE DE QUADROS CLÍNICOS: UM ENFOQUE DA PSICOFÍSICA CLÍNICA
}

\author{
Ricardo Kamizaki* \\ Fátima Aparecida Emm Faleiros Sousa** \\ Roberta P.M.Sant'ana*** \\ José Aparecido da Silva****
}

KAMIZAKI, R.; SOUSA, F.A.E.F., SANT’ANA, R.P.M., SILVA, J.A.da. Estimação de magnitude da gravidade de quadros clínicos: um enfoque da psicofísica clínica. Rev.latino-am.enfermagem, Ribeirão Preto, v. 7, n. 2, p. 55-62, abril 1999.

Neste experimento a gravidade de quadros clínicos foi avaliada através do método psicofisico de estimação de magnitudes. Os objetivos deste experimento foram: 1) Comparar as escalas derivadas de julgamentos de razão (estimativas de magnitudes) feitos por três diferentes amostras brasileiras; 2) Verificar a estabilidade e a concordância das estimativas da gravidade de quadros clínicos julgada no Brasil, E.U.A. e Inglaterra. Para tal foram convidados profissionais atuantes nas áreas de Psicologia, Enfermagem e Medicina. O coeficiente de correlação de Pearson entre os grupos foram de 0,92 a menor e 0,94 a maior. Os expoentes encontrados entre as amostras brasileira e americana foi de 1,22 e entre as amostras brasileira e inglesa foi de 1,15.

UNITERMOS: gravidade de enfermidades, estimação de magnitude, psicofisicica

Vários estudos demonstraram que a gravidade de quadros clínicos pode ser escalonada utilizando o método de estimação de magnitude, baseada em estimativas de amostras médicas e não médicas feitas por WYLER et al. (1968) e RIPPERE (1976), tendo como referência o prognóstico de morbidez do quadro clínico em questão.

A gravidade dos quadros clínicos (diagnósticos) contextualmente definida neste estudo foi como sendo o grau de severidade dos riscos de ameaça de vida ou de indicação de prognóstico ruim ou estado terminal.

Os estudos de WYLER et al. (1968) elaboraram uma lista de 126 doenças. Estes enviaram pelo correio os questionários para 300 participantes leigos e 150 médicos atuantes. A tarefa dos participantes, em ambas as amostras, consistia em estimar a magnitude da severidade das 126 doenças assinalando a cada uma delas um número que fosse proporcional ao valor 500 designado à doença Úlcera peptídica. Eis alguns exemplos das doenças listadas: astigmatismo, menopausa, coma, depressão, epilepsia, derrame cerebral, ataque cardíaco, uremia, câncer, leucemia, etc. As médias geométricas das estimativas de magnitude de cada uma das 126 doenças bem como suas respectivas ordenações foram calculadas para ambas as amostras de participantes. Um coeficiente de correlação de ordem de Spearman (rho) foi usado para comparar as ordenações dos dois grupos. Chegou-se ao resultado de rho $=0,95$, indicando uma alta concordância entre as estimativas de magnitude dos dois grupos.

Quanto ao grau de severidade comparativa entre as enfermidades verificou-se que a caspa obteve a menor estimativa de magnitude, o aborto obteve estimativa de magnitude média e a leucemia foi a doença com maior estimativa de magnitude. O mesmo padrão de resultados foi confirmado por WYLER et al.(1971) e por VOLICER \& BOHANSON (1975).

Em outro estudo similar, WYLER et al. (1970) replicaram o trabalho original usando uma nova amostra de médicos. Assim os resultados obtidos dos 117 participantes do estudo de 1968 foram comparados com os obtidos por uma nova amostra de 203 médicos exercendo diferentes especialidades clínicas. Um coeficiente de correlação de ordem de Spearman (rho) calculado a partir das ordenações de enfermidades feitas pelos dois grupos foi igual a 0,99 , indicando que os dois grupos foram homogêneos e consistentes em suas

\footnotetext{
* Aluno de doutorado da Faculdade de Filosofia, Ciências e Letras de Ribeirão Preto da Universidade de São Paulo

** Professor Associado do Departamento de Enfermagem Geral e Especializada da Escola de Enfermagem de Ribeirão Preto da Universidade de São Paulo

*** Aluna de Aperfeiçoamento da Escola de Enfermagem de Ribeirão Preto da Universidade de São Paulo

**** Professor Titular da Faculdade de Filosofia, Ciências e Letras de Ribeirão Preto da Universidade de São Paulo
} 
estimativas de magnitudes da severidade das 126 doenças. As estimativas feitas por médicos de diferentes especialidades não foram significativamente diferentes entre si, indicando, portanto, que a variável especialidade do respondente não é significativa.

RIPPERE (1976) fez um estudo sobre severidade de quadros clínicos utilizando escalas de razão e de categorias. Para tal, a autora utilizou 12 quadros clínicos (bronquite, luxação, úlcera estomacal, hipertensão, pneumonia, sífilis, tuberculose, diabetes, derrame, coágulo nos vasos, câncer e leucemia) dos 126 propostos por WYLER et al. (1968) e aplicou nesses participantes a estimação de magnitudes. A correlação de Spearman entre os participantes de WYLER et al. e RIPPERE foi de 0,89 , sendo portanto alta, positiva e significativa. Os dois métodos, estimação de categorias e de magnitudes, apontam para os mesmos resultados. $\mathrm{O}$ coeficiente de concordância de Kendall (W) foi de 0,97 ( $<<0,005)$, indicando uma alta ordenação entre as amostras.

Assim, optamos por fundamentar este trabalho utilizando os procedimentos usados na Psicofísica Sensorial e/ou Social e/ou Clínica (para detalhes ver SOUSA \& SILVA, 1996; STEVENS, 1975 e SOUSA, 1993) com o objetivo de: 1) Escalonar os quadros clínicos (diagnósticos) utilizados no estudo de WYLER et al. (1968). Selecionamos 98 quadros clínicos (diagnósticos) da lista original de WYLER et al. (1968), uma vez que retiramos alguns como, por exemplo, mordida de tubarão e acrescentamos outros como AIDS e coqueluche, com o propósito de adequação à nossa realidade e atualização dos quadros clínicos; 2) Validar a escala psicofísica de gravidade dos quadros clínicos.

Avaliação dos quadros clínicos através do método psicofísico escalar de estimação de magnitudes.

Neste experimento a gravidade de quadros clínicos foi avaliada através do método psicofísico de estimação de magnitudes. Os objetivos deste experimento foram: 1) Comparar as escalas derivadas de julgamentos de razão (estimativas de magnitudes) feitos por três diferentes amostras brasileiras; 2) Verificar a estabilidade e a concordância das estimativas da gravidade de quadros clínicos julgada no Brasil, E.U.A e Inglaterra.

\section{MÉTODO}

Participantes. Foram convidados 47 participantes, sendo 20 enfermeiros (Amostra A1), 7 psicólogos (Amostra A2) e 20 médicos (Amostra A3), sendo eles profissionais atuantes na cidade de Ribeirão Preto, com idades variando entre 23 a 75 anos. Todos eram ingênuos quanto aos propósitos do Experimento.

Material. Foi montado um bloco de papel contendo na primeira página instruções específicas para o método utilizado e nas páginas seguintes uma lista de 100 quadros clínicos (diagnósticos) e caneta.

Procedimento. O método escalar utilizado foi o de estimação de magnitudes. A tarefa dos participantes foi assinalar um número a cada quadro clínico (diagnóstico) que fosse proporcional a quantidade de gravidade dos quadros clínicos. Dessa forma, se o participante julgasse que um dado clínico possui duas vezes mais gravidade do que um outro quadro clínico, ele deveria assinalar a ele um número duas vezes maior. Se ele julgasse que um dado quadro clínico possui metade da gravidade do que um outro quadro clínico, ele deveria assinalar a ele um número que fosse metade do atribuído aquele quadro clínico. Os diferentes quadros clínicos foram apresentados em uma série de 100, os quais foram dispostos, em cada página contendo em média vinte quadros clínicos, numa ordem totalmente aleatória para todos participantes. Cada participante estabeleceu 100 estimativas, sendo uma para cada quadro clínico. Estímulo padrão e módulo foram designados previamente, tendo sido estabelecido o valor de 100 para Úlcera peptídica.

As instruções dadas para os participantes requeriam que os julgamentos fossem realizados em termos do grau relativo de gravidades dos quadros clínicos, considerando-se a maioria dos pacientes, cujo problema central fosse esse quadro clínico e as outras condições relativamente controladas. Todos os participantes fizeram a tarefa ora em laboratório, ora em suas residências, ora em locais de trabalho, sendo que, o instrumento era entregue e posteriormente recolhido, após serem instruídos a respondê-lo individualmente e sem interrupção. Adicionado a isso foram instruídos a colocar a letra D no espaço correspondente às estimativas, caso não conhecesse algum diagnóstico listado.

\section{RESULTADOS E DISCUSSÃO}

As estimativas médias para os diferentes quadros clínicos realizados pelos diferentes grupos de participantes (A1 amostra composta por enfermeiros, A2 por psicólogos e A3 por médicos) e as respectivas ordenações de posições estão apresentadas na Tabela 1.

Pode-se observar na Tabela 1 que os quadros clínicos caspa, verruga, terçol, acne e ferida comum foram considerados os de menor gravidade, enquanto que, os quadros clínicos AIDS, câncer, leucemia, coma e hemorragia cerebral foram os de maior gravidade para as três amostras brasileiras. De fato, o coeficiente de concordância de Kendall (W) mostrou que, as diferentes amostras brasileiras foram altamente concordantes quanto à ordenação desses diferentes quadros clínicos (diagnósticos). Esse valor do coeficiente de concordância foi $\mathrm{W}=0,96(\mathrm{p}<0,001)$. 
Tabela 1 - Média geométrica das estimativas de magnitudes das amostras brasileiras compostas de Enfermeiros (EM1), Psicólogos (EM2), Médicos (EM3) e americanas compostas de Médicos (EMM), Leigos (EML), média geométrica geral das amostras brasileiras (EMB) e média geral americana (EMA) e respectivas ordenações de posições (OP) das gravidades julgadas de cada quadro clínico

\begin{tabular}{|c|c|c|c|c|c|c|c|c|c|c|c|c|c|c|}
\hline Diagnóstico & EM1 & OP & EM2 & OP & EM3 & OP & EMB & OP & EMM & OP & EML & OP & EMA & OP \\
\hline Caspa & 16,48 & $2^{o}$ & 1,90 & $1^{\mathrm{o}}$ & 17,83 & $1^{\mathrm{o}}$ & 12,36 & $1^{\mathrm{o}}$ & 2,80 & $1^{\mathrm{o}}$ & 2,00 & $1^{\mathrm{o}}$ & 2,37 & $1^{\mathrm{o}}$ \\
\hline Verruga & 16,52 & $3^{\circ}$ & 3,79 & $2^{\circ}$ & 29,41 & $2^{\circ}$ & 16,96 & $2^{\circ}$ & 4,00 & $3^{\circ}$ & 6,00 & $3^{\circ}$ & 4,90 & $3^{\circ}$ \\
\hline Terçol & 16,31 & $1^{\circ}$ & 8,08 & $6^{\circ}$ & 35,18 & $4^{\circ}$ & 20,48 & $3^{\circ}$ & 10,60 & $7^{\circ}$ & 10,60 & $7^{\circ}$ & 10,60 & $7^{\circ}$ \\
\hline Acne & 17,69 & $4^{\circ}$ & 7,28 & $4^{\circ}$ & 34,48 & $3^{\circ}$ & 20,59 & $4^{o}$ & 23,00 & $18^{\circ}$ & 24,00 & $18^{\circ}$ & 23,49 & $18^{\circ}$ \\
\hline Ferida Comum & 30,63 & $9^{\circ}$ & 7,63 & $5^{\circ}$ & 35,58 & $6^{\circ}$ & 26,54 & $5^{\circ}$ & 7,00 & $2^{\circ}$ & 3,40 & $2^{\circ}$ & 4,88 & $2^{\circ}$ \\
\hline Período Pré-Menstrual & 20,87 & $5^{\circ}$ & 26,15 & $16^{\circ}$ & 35,40 & $5^{\circ}$ & 27,04 & $6^{\circ}$ & 29,20 & $24^{\circ}$ & 31,00 & $25^{\circ}$ & 30,00 & $25^{\circ}$ \\
\hline Resfriado & 25,95 & $8^{\circ}$ & 10,60 & $8^{\circ}$ & 42,31 & $7^{\circ}$ & 28,00 & $7^{\circ}$ & 10,20 & $4^{\circ}$ & 10,00 & $4^{\circ}$ & 10,00 & $4^{\circ}$ \\
\hline Astigmatismo & 34,40 & $11^{\circ}$ & 6,06 & $3^{\circ}$ & 45,02 & $10^{\circ}$ & 29,79 & $8^{\circ}$ & 14,20 & $8^{\circ}$ & 12,80 & $10^{\circ}$ & 13,48 & $10^{\circ}$ \\
\hline Menopausa & 24,87 & $7^{\mathrm{o}}$ & 20,76 & $14^{\circ}$ & 51,02 & $11^{\circ}$ & 32,88 & $9^{\circ}$ & 24,60 & $13^{\circ}$ & 18,60 & $15^{\circ}$ & 21,39 & $15^{\circ}$ \\
\hline Furúnculo & 38,60 & $12^{\circ}$ & 11,77 & $9^{\circ}$ & 42,53 & $8^{\circ}$ & 33,70 & $10^{\circ}$ & 17,80 & $12^{\circ}$ & 18,40 & $11^{\circ}$ & 18,10 & $11^{\circ}$ \\
\hline Verminose & 39,27 & $13^{\circ}$ & 16,26 & $11^{\circ}$ & 44,84 & $9^{\circ}$ & 36,43 & $11^{\circ}$ & 13,00 & $10^{\circ}$ & 13,20 & $9^{\circ}$ & 13,10 & $9^{\circ}$ \\
\hline Miopia & 51,40 & $21^{\circ}$ & 8,26 & $7^{\circ}$ & 66,82 & $16^{\circ}$ & 43,62 & $12^{\circ}$ & 13,20 & $9^{\circ}$ & 12,80 & $8^{\circ}$ & 13,00 & $8^{\circ}$ \\
\hline Amidalite & 43,27 & $16^{\circ}$ & 27,37 & $18^{\circ}$ & 51,91 & $12^{\circ}$ & 43,68 & $13^{\circ}$ & 17,40 & $14^{\circ}$ & 21,80 & $13^{\circ}$ & 19,48 & $13^{\circ}$ \\
\hline Reação Ansiosa & 32,73 & $10^{\circ}$ & 52,77 & $38^{\circ}$ & 56,63 & $13^{\circ}$ & 44,51 & $14^{\circ}$ & 70,00 & $46^{\circ}$ & 60,20 & $47^{\circ}$ & 64,92 & $47^{\circ}$ \\
\hline Sinusite & 43,65 & $17^{\circ}$ & 22,59 & $15^{\circ}$ & 60,55 & $14^{\circ}$ & 45,48 & $15^{\circ}$ & 29,20 & $31^{\circ}$ & 35,60 & $28^{\circ}$ & 32,24 & $28^{\circ}$ \\
\hline Ulceração pelo Frio & 40,78 & $15^{\circ}$ & 26,67 & $17^{\circ}$ & 67,34 & $17^{\circ}$ & 48,07 & $16^{\circ}$ & 47,60 & $32^{\circ}$ & 40,40 & $37^{\circ}$ & 43,85 & $37^{\circ}$ \\
\hline Desvio Sexual & 24,13 & $6^{\circ}$ & 47,85 & $30^{\circ}$ & 124,60 & $52^{\circ}$ & 49,73 & $17^{\circ}$ & 99,00 & $47^{\circ}$ & 66,20 & $54^{\circ}$ & 80,96 & $54^{\circ}$ \\
\hline Luxação & 40,27 & $14^{\circ}$ & 16,01 & $10^{\circ}$ & 98,61 & $41^{\circ}$ & 51,39 & $18^{\circ}$ & 92,20 & $67^{\circ}$ & 110,20 & $65^{\circ}$ & 100,80 & $65^{\circ}$ \\
\hline Carbúnculo & 45,12 & $18^{\circ}$ & 20,00 & $12^{\circ}$ & 66,60 & $15^{\circ}$ & 55,15 & $19^{\circ}$ & 22,60 & $16^{\circ}$ & 23,40 & $17^{\circ}$ & 23,00 & $17^{\mathrm{o}}$ \\
\hline Cachumba & 57,92 & $24^{\circ}$ & 31,57 & $21^{\circ}$ & 68,67 & $18^{\circ}$ & 56,66 & $20^{\circ}$ & 22,60 & $21^{\circ}$ & 27,60 & $20^{\circ}$ & 24,98 & $19^{\circ}$ \\
\hline Catapora & 60,31 & $25^{\circ}$ & 37,28 & $24^{\circ}$ & 72,61 & $21^{\circ}$ & 60,27 & $21^{\circ}$ & 21,00 & $17^{\circ}$ & 23,80 & $16^{\mathrm{o}}$ & 22,36 & $16^{\circ}$ \\
\hline Infecção de Ouvido Médio & 54,61 & $22^{\circ}$ & 41,20 & $27^{\circ}$ & 76,67 & $25^{\circ}$ & 60,64 & $22^{\circ}$ & 27,20 & $26^{\circ}$ & 31,20 & $23^{\circ}$ & 29,13 & $23^{\circ}$ \\
\hline Insolação & 50,79 & $20^{\circ}$ & 47,24 & $29^{\circ}$ & 81,64 & $28^{\circ}$ & 61,49 & $23^{\circ}$ & 10,80 & $5^{\circ}$ & 10,20 & $5^{\circ}$ & 10,50 & $5^{o}$ \\
\hline Hérnia & 66,40 & $32^{\circ}$ & 28,69 & $19^{\circ}$ & 78,30 & $27^{\circ}$ & 62,86 & $24^{\circ}$ & 32,60 & $35^{\circ}$ & 41,00 & $29^{\circ}$ & 36,56 & $29^{\circ}$ \\
\hline Enxaqueca & 56,21 & $23^{\circ}$ & 53,85 & $40^{\circ}$ & 77,62 & $26^{\circ}$ & 64,07 & $25^{\circ}$ & 48,00 & $39^{\circ}$ & 47,60 & $40^{\circ}$ & 47,80 & $40^{\circ}$ \\
\hline Daltonismo & 76,90 & $44^{\circ}$ & 30,12 & $20^{\circ}$ & 69,85 & $19^{\circ}$ & 64,08 & $26^{\circ}$ & 19,20 & $11^{\circ}$ & 18,20 & $12^{\circ}$ & 18,69 & $12^{\circ}$ \\
\hline Ferida do Útero & 65,37 & $29^{\circ}$ & 39,15 & $25^{\circ}$ & 84,47 & $30^{\circ}$ & 67,40 & $27^{\circ}$ & 28,00 & $26^{\circ}$ & 31,00 & $24^{\circ}$ & 29,46 & $24^{\circ}$ \\
\hline Coqueluche & 62,90 & $26^{\circ}$ & 63,35 & $44^{\circ}$ & 75,77 & $24^{\circ}$ & 68,16 & $28^{\circ}$ & - & - & - & - & - & - \\
\hline Hemorragia & 66,08 & $30^{\circ}$ & 54,88 & $42^{\circ}$ & 83,66 & $29^{\circ}$ & 70,81 & $29^{\circ}$ & 26,00 & $19^{\circ}$ & 24,20 & $19^{\circ}$ & 25,08 & $20^{\circ}$ \\
\hline Bursite & 66,09 & $31^{\circ}$ & 49,59 & $32^{\circ}$ & 85,27 & $31^{\circ}$ & 71,41 & $30^{\circ}$ & 34,80 & $33^{\circ}$ & 40,40 & $33^{\circ}$ & 37,50 & $30^{\circ}$ \\
\hline Esquizofrenia & 64,78 & $28^{\circ}$ & 100,40 & $55^{\circ}$ & 73,34 & $23^{\circ}$ & 72,04 & $31^{\circ}$ & 43,60 & $29^{\circ}$ & 32,60 & $36^{\circ}$ & 37,70 & $31^{\circ}$ \\
\hline Anemia & 81,40 & $50^{\circ}$ & 50,52 & $33^{\circ}$ & 72,76 & $22^{\circ}$ & 72,28 & $32^{\circ}$ & 56,00 & $38^{\circ}$ & 47,40 & $38^{\circ}$ & 51,52 & $43^{\circ}$ \\
\hline Sarampo & 69,27 & $36^{\circ}$ & 52,09 & $37^{\circ}$ & 87,39 & $35^{\circ}$ & 73,38 & $33^{\circ}$ & 24,80 & $22^{\circ}$ & 28,20 & $22^{\circ}$ & 26,45 & $21^{\circ}$ \\
\hline Bócio & 67,83 & $33^{\circ}$ & 40,72 & $26^{\circ}$ & 94,83 & $39^{\circ}$ & 73,97 & $34^{\circ}$ & 50,40 & $41^{\circ}$ & 50,40 & $41^{\circ}$ & 50,40 & $41^{\circ}$ \\
\hline Olho infectado & 71,85 & $40^{\circ}$ & 50,52 & $34^{\circ}$ & 88,62 & $36^{\circ}$ & 74,27 & $35^{\circ}$ & 45,20 & $28^{\circ}$ & 32,40 & $28^{\circ}$ & 38,27 & $33^{\circ}$ \\
\hline Veia Varicosa & 71,99 & $42^{\circ}$ & 53,13 & $39^{\circ}$ & 85,44 & $33^{\circ}$ & 76,33 & $36^{\circ}$ & 29,40 & $20^{\circ}$ & 27,40 & $21^{\circ}$ & 28,38 & $22^{\circ}$ \\
\hline Absesso Dental & 69,08 & $35^{\circ}$ & 51,19 & $35^{\circ}$ & 98,93 & $42^{\circ}$ & 77,16 & $37^{\circ}$ & 18,60 & $15^{\circ}$ & 22,00 & $15^{\circ}$ & 20,23 & $14^{\mathrm{o}}$ \\
\hline Psoriase & 69,30 & $37^{\circ}$ & 36,84 & $23^{\circ}$ & 96,20 & $40^{\circ}$ & 77,24 & $38^{\circ}$ & 41,00 & $40^{\circ}$ & 50,20 & $40^{\circ}$ & 45,37 & $38^{\circ}$ \\
\hline Mononucleose & 68,76 & $34^{\circ}$ & 80,00 & $48^{\circ}$ & 85,29 & $32^{\circ}$ & 79,21 & $39^{\circ}$ & 28,40 & $26^{\circ}$ & 32,00 & $27^{\circ}$ & 30,15 & $26^{\circ}$ \\
\hline Gonorréia & 82,32 & $52^{\circ}$ & 97,03 & $53^{\circ}$ & 71,85 & $20^{\circ}$ & 79,62 & $40^{\circ}$ & 36,00 & $27^{\circ}$ & 40,80 & $34^{\circ}$ & 38,32 & $34^{\circ}$ \\
\hline Pressão Baixa & 79,03 & $46^{\circ}$ & 64,92 & $45^{\circ}$ & 86,88 & $34^{\circ}$ & 79,76 & $41^{\circ}$ & 19,60 & $44^{\circ}$ & 56,20 & $44^{\circ}$ & 37,80 & $32^{\circ}$ \\
\hline Frigidez & 46,98 & $19^{\circ}$ & 77,10 & $46^{\circ}$ & 134,27 & $57^{\circ}$ & 79,97 & $42^{\circ}$ & 71,60 & $54^{\circ}$ & 82,00 & $54^{\circ}$ & 76,62 & $51^{\circ}$ \\
\hline Cisto Ovariano & 71,89 & $41^{\circ}$ & 48,80 & $31^{\circ}$ & 107,85 & $46^{\circ}$ & 81,53 & $43^{\circ}$ & 47,60 & $37^{\circ}$ & 46,00 & $39^{\circ}$ & 46,79 & $39^{\circ}$ \\
\hline Artrite & 63,94 & $27^{\circ}$ & 45,94 & $28^{\circ}$ & 130,14 & $55^{\circ}$ & 82,36 & $44^{\circ}$ & 101,40 & $63^{\circ}$ & 94,20 & $63^{\circ}$ & 97,73 & $63^{\circ}$ \\
\hline Gota & 69,67 & $38^{\circ}$ & 51,72 & $36^{\circ}$ & 122,86 & $51^{\circ}$ & 84,84 & $45^{\circ}$ & 50,40 & $42^{\circ}$ & 50,40 & $42^{\circ}$ & 50,40 & $42^{\circ}$ \\
\hline Varíola & 73,05 & $43^{\circ}$ & 55,64 & $43^{\circ}$ & 119,39 & $50^{\circ}$ & 85,23 & $46^{\circ}$ & 133,00 & $74^{\circ}$ & 131,40 & $74^{\circ}$ & 132,20 & $73^{\circ}$ \\
\hline Herpes & 78,51 & $45^{\circ}$ & 79,53 & $47^{\circ}$ & 106,61 & $45^{\circ}$ & 90,10 & $47^{\circ}$ & 41,60 & $30^{\circ}$ & 35,40 & $30^{\circ}$ & 38,37 & $35^{\circ}$ \\
\hline Surdez & 80,01 & $48^{\circ}$ & 36,31 & $22^{\circ}$ & 148,14 & $62^{\circ}$ & 92,45 & $48^{\circ}$ & 105,20 & $70^{\circ}$ & 121,40 & $70^{\circ}$ & 113,01 & $69^{\circ}$ \\
\hline Asma & 80,48 & $49^{\circ}$ & 54,56 & $41^{\circ}$ & 130,12 & $54^{\circ}$ & 93,48 & $49^{\circ}$ & 91,40 & $57^{\circ}$ & 89,00 & $57^{\circ}$ & 90,19 & $59^{\circ}$ \\
\hline Bronquite & 91,60 & $57^{\circ}$ & 89,72 & $51^{\circ}$ & 100,51 & $44^{\circ}$ & 94,88 & $50^{\circ}$ & 37,20 & $36^{\circ}$ & 44,20 & $36^{\circ}$ & 40,55 & $36^{\circ}$ \\
\hline Queimadura & 95,88 & $60^{\circ}$ & 117,81 & $61^{\circ}$ & 94,28 & $38^{\circ}$ & 98,30 & $51^{\circ}$ & 82,80 & $52^{\circ}$ & 76,00 & $52^{\circ}$ & 79,33 & $52^{\circ}$ \\
\hline Pedra na Vesícula & 87,25 & $54^{\circ}$ & 102,64 & $58^{\circ}$ & 110,73 & $47^{\circ}$ & 99,20 & $52^{\circ}$ & 82,80 & $59^{\circ}$ & 90,40 & $59^{\circ}$ & 86,52 & $57^{\circ}$ \\
\hline Febre de Feno & 102,52 & $62^{\circ}$ & 223,61 & $87^{\circ}$ & 89,12 & $37^{\circ}$ & 99,92 & $53^{\circ}$ & 32,80 & $23^{\circ}$ & 28,40 & $23^{\circ}$ & 30,52 & $27^{\circ}$ \\
\hline Úlcera peptídica & 100,00 & $61^{\circ}$ & 100,00 & $54^{\circ}$ & 100,00 & $43^{\circ}$ & 100,00 & $54^{\circ}$ & 100,00 & $65^{\circ}$ & 100,00 & $65^{\circ}$ & 100,00 & $64^{\circ}$ \\
\hline Obesidade & 79,84 & $47^{\circ}$ & 96,86 & $52^{\circ}$ & 126,51 & $53^{\circ}$ & 100,44 & $55^{\circ}$ & 70,20 & $48^{\circ}$ & 69,00 & $48^{\circ}$ & 69,60 & $49^{\circ}$ \\
\hline Pneumonia & 91,30 & $56^{\circ}$ & 119,38 & $62^{\circ}$ & 113,67 & $48^{\circ}$ & 104,31 & $56^{\circ}$ & 59,60 & $45^{\circ}$ & 57,20 & $45^{\circ}$ & 58,39 & $45^{\circ}$ \\
\hline Hipertensão & 81,78 & $51^{\circ}$ & 108,37 & $59^{\circ}$ & 133,24 & $56^{\circ}$ & 104,92 & $57^{\circ}$ & 91,20 & $61^{\circ}$ & 92,00 & $61^{\circ}$ & 91,60 & $60^{\circ}$ \\
\hline Apendicite & 85,01 & $53^{\circ}$ & 84,20 & $49^{\circ}$ & 149,50 & $63^{\circ}$ & 108,50 & $58^{\circ}$ & 59,60 & $53^{\circ}$ & 78,20 & $53^{\circ}$ & 68,27 & $48^{\circ}$ \\
\hline Glaucoma & 70,62 & $39^{\circ}$ & 101,11 & $56^{\circ}$ & 173,30 & $70^{\circ}$ & 109,16 & $59^{\circ}$ & 79,60 & $58^{\circ}$ & 89,20 & $58^{\circ}$ & 84,26 & $56^{\circ}$ \\
\hline Esgotamento Nervoso & 107,95 & $66^{\circ}$ & 109,77 & $60^{\circ}$ & 116,32 & $49^{\circ}$ & 111,80 & $60^{\circ}$ & 120,20 & $64^{\circ}$ & 99,00 & $64^{\circ}$ & 109,09 & $66^{\circ}$ \\
\hline Depressão & 92,59 & $58^{\circ}$ & 136,08 & $65^{\circ}$ & 137,41 & $58^{\circ}$ & 115,99 & $61^{\circ}$ & 88,20 & $51^{\circ}$ & 73,40 & $51^{\circ}$ & 80,46 & $53^{\circ}$ \\
\hline Impotência Sexual & 107,03 & $65^{\circ}$ & 101,11 & $57^{\circ}$ & 143,10 & $59^{\circ}$ & 120,08 & $62^{\circ}$ & 79,60 & $56^{\circ}$ & 84,60 & $56^{\circ}$ & 82,06 & $55^{\circ}$ \\
\hline Pressão Alta & 93,04 & $59^{\circ}$ & 126,07 & $63^{\circ}$ & 167,70 & $68^{\circ}$ & 125,89 & $63^{\circ}$ & 114,20 & $69^{\circ}$ & 120,60 & $69^{\circ}$ & 117,36 & $70^{\circ}$ \\
\hline Sífilis & 105,97 & $64^{\circ}$ & 151,12 & $70^{\circ}$ & 145,84 & $61^{\circ}$ & 128,51 & $64^{\circ}$ & 74,40 & $50^{\circ}$ & 70,40 & $50^{\circ}$ & 72,37 & $50^{\circ}$ \\
\hline Uremia & 87,76 & $55^{\circ}$ & 20,00 & $13^{\circ}$ & 210,96 & $82^{\circ}$ & 131,12 & $65^{\circ}$ & 218,00 & $98^{\circ}$ & 218,00 & $98^{\circ}$ & 218,00 & $98^{\circ}$ \\
\hline
\end{tabular}


Tabela 1 (continuação)

\begin{tabular}{|c|c|c|c|c|c|c|c|c|c|c|c|c|c|c|}
\hline Diagnóstico & EMI & $\mathbf{O P}$ & EM2 & $\mathbf{O P}$ & EM3 & $\mathbf{O P}$ & EMB & $\mathbf{O P}$ & EMM & $\mathbf{O P}$ & EML & $\mathbf{O P}$ & EMA & $\mathbf{O P}$ \\
\hline Queimadura do Sol & 120,81 & $72^{\circ}$ & 87,22 & $50^{\circ}$ & 164,99 & $67^{\circ}$ & 131,49 & $66^{\circ}$ & 10,80 & $6^{\circ}$ & 10,20 & $6^{\circ}$ & 10,50 & $6^{8}$ \\
\hline Erveneramento Acidental & 112,27 & $68^{\circ}$ & 156,02 & $71^{\circ}$ & 157,01 & $65^{\circ}$ & 136,00 & $67^{\circ}$ & 91,80 & $55^{\circ}$ & 82,00 & $55^{\circ}$ & 86,76 & $58^{\circ}$ \\
\hline Mal de Parkinson & 11193 & $67^{\circ}$ & 170,21 & $76^{\circ}$ & 169,19 & $\theta 9^{\circ}$ & 140,92 & $68^{\circ}$ & 141,00 & $81^{\circ}$ & 146,80 & $80^{\circ}$ & 143,87 & $76^{\circ}$ \\
\hline Distrofia Muscular & 103,24 & $63^{\circ}$ & 157,04 & $72^{\circ}$ & 206,69 & $78^{\circ}$ & 146,36 & $69^{\circ}$ & 164,00 & $93^{\circ}$ & $167, \infty$ & $93^{\circ}$ & 165,49 & $91^{\circ}$ \\
\hline Twerculose & 132,93 & $78^{\circ}$ & 139,35 & $66^{\circ}$ & 177,02 & $72^{\circ}$ & 150,71 & $70^{\circ}$ & 122,60 & $71^{\circ}$ & 121,40 & $71^{\circ}$ & 122,00 & $71^{\circ}$ \\
\hline Insuficiência Renal & 155,13 & $87^{\circ}$ & 168,02 & $75^{\circ}$ & 143,62 & $60^{\circ}$ & 151,85 & $71^{\circ}$ & 59,60 & $49^{\circ}$ & 70,20 & $49^{\circ}$ & 64,68 & $46^{\circ}$ \\
\hline Diabetes & 119,14 & $71^{\circ}$ & 142,62 & $68^{\circ}$ & 199,89 & $77^{\circ}$ & 152,52 & $72^{\circ}$ & 135,00 & $73^{\circ}$ & 130,40 & $73^{\circ}$ & 132,68 & $74^{\circ}$ \\
\hline Epilepsia & 118,19 & $70^{\circ}$ & 142,87 & $9^{\circ}$ & 208,83 & $80^{\circ}$ & 154,90 & $73^{\circ}$ & 112,80 & $68^{\circ}$ & 112,40 & $68^{\circ}$ & 112,60 & $68^{\circ}$ \\
\hline Coágulo Vasos Sanguíneos & 147,32 & $84^{\circ}$ & 18860 & $80^{\circ}$ & 155,12 & $64^{\circ}$ & 15578 & $74^{\circ}$ & 164,00 & $7^{\circ}$ & 14260 & $7^{\circ}$ & 15293 & $82^{\circ}$ \\
\hline Endurecimento das Artérias & 128,52 & $75^{\circ}$ & 187,54 & $79^{\circ}$ & 177,21 & $73^{\circ}$ & 155,88 & $75^{\circ}$ & 120,40 & $66^{\circ}$ & 103,80 & $66^{\circ}$ & 111,79 & $67^{\circ}$ \\
\hline Colapso Pulmonar & 146,68 & $83^{\circ}$ & 195,57 & $82^{\circ}$ & 158,24 & $66^{\circ}$ & 158,12 & $76^{\circ}$ & 90,00 & $62^{\circ}$ & 93,60 & $62^{\circ}$ & 91,78 & $61^{\circ}$ \\
\hline Adição de Drogas & 144,49 & $82^{\circ}$ & 158,56 & $73^{\circ}$ & 177,81 & $74^{\circ}$ & 160,38 & $7^{\circ}$ & 147,00 & $79^{\circ}$ & $144, \infty 0$ & $79^{\circ}$ & 145,90 & $77^{\circ}$ \\
\hline Hepatite & 141,79 & $81^{\circ}$ & 140,16 & $67^{\circ}$ & 191,79 & $76^{\circ}$ & 160,96 & $78^{\circ}$ & 97,00 & $60^{\circ}$ & 90,40 & $60^{\circ}$ & 93,64 & $62^{\circ}$ \\
\hline Enfisema & 123,07 & $73^{\circ}$ & 163,21 & $74^{\circ}$ & 208,41 & $79^{\circ}$ & 161,50 & $79^{\circ}$ & 137,40 & $75^{\circ}$ & 136,40 & $75^{\circ}$ & 136,90 & $75^{\circ}$ \\
\hline Psicose Maríco-Depressiva & 131,45 & $76^{\circ}$ & 249,80 & $91^{\circ}$ & 176,03 & $71^{\circ}$ & 163,05 & $80^{\circ}$ & 160,00 & $89^{\circ}$ & $161, \infty$ & $89^{\circ}$ & 160,90 & $90^{\circ}$ \\
\hline Mordidade Cobra & 132,67 & $77^{\circ}$ & 129,40 & $64^{\circ}$ & 222,70 & $84^{\circ}$ & $163, \pi 0$ & $81^{\circ}$ & 64,40 & $43^{\circ}$ & 52,00 & $44^{\circ}$ & 57,87 & $44^{\circ}$ \\
\hline Esquizofienia & 128,29 & $74^{\circ}$ & 177,84 & $7^{\circ}$ & 219,91 & $83^{\circ}$ & 169,40 & $82^{\circ}$ & 170,40 & $92^{\circ}$ & 166,80 & $92^{\circ}$ & 168,59 & $93^{\circ}$ \\
\hline Cegueira & 114,34 & $69^{\circ}$ & 211,93 & $84^{\circ}$ & 227,85 & $86^{\circ}$ & 169,51 & $83^{\circ}$ & 148,40 & $90^{\circ}$ & 162,20 & $90^{\circ}$ & 155,15 & $85^{\circ}$ \\
\hline Pancreatite & 136,91 & $80^{\circ}$ & 187,23 & $78^{\circ}$ & 210,73 & $81^{\circ}$ & 171,58 & $84^{\circ}$ & 124,80 & $72^{\circ}$ & 121,40 & $72^{\circ}$ & 123,00 & $72^{\circ}$ \\
\hline Coágulo nos Pulmões & 183,00 & $91^{\circ}$ & 239,35 & $8^{\circ}$ & 178,17 & $75^{\circ}$ & 188,57 & $85^{\circ}$ & 164,00 & $78^{\circ}$ & 142,60 & $78^{\circ}$ & 152,93 & $83^{\circ}$ \\
\hline Problema Card Congênito & 156,19 & $89^{\circ}$ & 195,20 & $81^{\circ}$ & 233,23 & $87^{\circ}$ & 191,50 & $86^{\circ}$ & 167,00 & $82^{\circ}$ & 150,20 & $82^{\circ}$ & 158,38 & $8^{\circ}$ \\
\hline Retardo Mental & 148,65 & $85^{\circ}$ & 239,35 & $89^{\circ}$ & 227,67 & $85^{\circ}$ & 192,37 & $87^{\circ}$ & 150,40 & $91^{\circ}$ & 164,20 & $91^{\circ}$ & 157,15 & $87^{\circ}$ \\
\hline Esclerose & 136,30 & $79^{\circ}$ & 220,93 & $86^{\circ}$ & 253,35 & $89^{\circ}$ & 194,80 & $8^{\circ}$ & 182,00 & $94^{\circ}$ & 172,60 & $94^{\circ}$ & 177,24 & $94^{\circ}$ \\
\hline Derrame & 155,99 & $8^{\circ}$ & 211,21 & $83^{\circ}$ & 244,01 & $88^{\circ}$ & 198,43 & $89^{\circ}$ & 153,20 & $85^{\circ}$ & $151, \infty$ & $85^{\circ}$ & 152,50 & $81^{\circ}$ \\
\hline Cirrose Hepática & 154,27 & $86^{\circ}$ & 21879 & $85^{\circ}$ & 270,74 & $91^{\circ}$ & 206,46 & $90^{\circ}$ & 145,00 & $81^{\circ}$ & 148,80 & $81^{\circ}$ & 146,89 & $78^{\circ}$ \\
\hline Irfecçäo Cerebral & 184,67 & $92^{\circ}$ & 300,00 & $93^{\circ}$ & 270,36 & $90^{\circ}$ & 233,38 & $91^{\circ}$ & 177,00 & $88^{\circ}$ & 159,60 & $8^{\circ}$ & 168,07 & $92^{\circ}$ \\
\hline Ataque Cardíaco & 178,47 & $90^{\circ}$ & 309,17 & $94^{\circ}$ & 282,75 & $92^{\circ}$ & 237,01 & $92^{\circ}$ & 170,00 & $86^{\circ}$ & $151, \infty$ & $86^{\circ}$ & 160,64 & $80^{\circ}$ \\
\hline Tumor & 193,00 & $94^{\circ}$ & 239,35 & $90^{\circ}$ & 299,75 & $93^{\circ}$ & 241,54 & $93^{\circ}$ & 162,20 & $83^{\circ}$ & 151,60 & $83^{\circ}$ & 156,81 & $8^{\circ}$ \\
\hline ParalisiaCerebral & 185,05 & $93^{\circ}$ & 322,14 & $95^{\circ}$ & 322,39 & $95^{\circ}$ & 256,30 & $94^{\circ}$ & 151,80 & $87^{\circ}$ & 156,60 & $87^{\circ}$ & 154,18 & $84^{\circ}$ \\
\hline Falência Cardíaca & 196,63 & $95^{\circ}$ & 276,82 & $92^{\circ}$ & 337,04 & $96^{\circ}$ & 258,39 & $95^{\circ}$ & 154,00 & $76^{\circ}$ & 141,20 & $76^{\circ}$ & 147,84 & $79^{\circ}$ \\
\hline Hemorragia Cerebral & 204,72 & $97^{\circ}$ & 336,25 & $97^{\circ}$ & 300,31 & $94^{\circ}$ & 258,64 & $96^{\circ}$ & 176,00 & $93^{\circ}$ & 206,00 & $96^{\circ}$ & 190,41 & $95^{\circ}$ \\
\hline Coma & 203,33 & $96^{\circ}$ & 324,02 & $96^{\circ}$ & 385,06 & $100^{\circ}$ & 284,15 & $97^{\circ}$ & 152,60 & $84^{\circ}$ & 151,60 & $84^{\circ}$ & 152,10 & $80^{\circ}$ \\
\hline Leucerria & 207,60 & $100^{\circ}$ & 363,82 & $98^{\circ}$ & 371,28 & $98^{\circ}$ & 289,04 & $98^{\circ}$ & 232,00 & $95^{\circ}$ & 177,40 & $95^{\circ}$ & 20287 & $96^{\circ}$ \\
\hline Cânoer & & $98^{\circ}$ & 389,00 & $99^{\circ}$ & 373,47 & $99^{\circ}$ & 292,02 & $99^{\circ}$ & 210,00 & $97^{\circ}$ & 216,20 & $97^{\circ}$ & 213,08 & $97^{\circ}$ \\
\hline AIDS & 206,89 & $99^{\circ}$ & 572,51 & $100^{\circ}$ & 337,77 & $97^{\circ}$ & 295,76 & $100^{\circ}$ & - & - & - & - & - & - \\
\hline
\end{tabular}

Obs: As médias geométricas das amostras AM e A1 foram divididas por 5, pois no estudo de WYLER et al. (1968) foi adotado o módulo 500 para Úlcera peptídica, enquanto que em nosso estudo foi 100

Como conseqüência dessa alta concordância dos julgamentos feitos pelas diferentes amostras brasileiras, as estimativas de todos os participantes foram agrupadas.

Essas estimativas juntamente com suas respectivas ordenações estão apresentadas na coluna EMB da Tabela 1. Pode-se observar nitidamente que, estas ordenações não foram substancialmente diferentes daquelas produzidas pelas amostras americanas, conforme também está apresentado na coluna EMA da Tabela 2.

Do mesmo modo, caspa, verruga, terçol, acne e ferida comum foram os quadros clínicos (diagnósticos) considerados os de menor gravidade para os brasileiros e caspa, verruga, ferida comum, resfriado e insolação para os americanos, enquanto que AIDS, câncer, leucemia, coma e hemorragia cerebral foram considerados os de maior gravidade para os brasileiros e uremia, câncer, leucemia, hemorragia cerebral e esclerose para os americanos. Um coeficiente de concordância aplicado às estimativas efetuadas pelas amostras gerais brasileiras e americanas, para os diferentes quadros clínicos mostrou $\mathrm{W}=0,95(\mathrm{p}<0,001)$, indicando portanto, que a ordenação por postos derivadas das estimativas de cada cultura (brasileira e americana) são altamente concordantes. Por exemplo, o quadro clínico caspa ocupa a $1^{\mathrm{a}}$ posição nas duas culturas e o quadro clínico adição de drogas a $77^{\mathrm{a}}$ posição nas duas culturas.

Os diagnósticos cujas estimativas de magnitude dos participantes brasileiros foram consideravelmente diferentes dos participantes do experimento de WYLER et al. (1968) foram: pressão baixa 79,62/37,80; veia varicosa $76,36 / 28,38$; febre do feno $99,92 / 30,52$; bronquite $94,88 / 40,55$; queimadura de sol profunda 131,49/10,50; mordida de cobra 163,70/57,87; falência cardíaca 258,39/147,84; tumor na coluna espinhal 241,54/ 156,81; paralisia cerebral 256,30/154,18 e hemorragia cerebral $258,64 / 190,41$. É interessante notar que os primeiros valores são médias geométricas dos participantes brasileiros, e estes tendem a superestimar os quadros clínicos apresentados em relação aos participantes americanos, talvez devido a aspectos 
culturais provenientes das diferenças entre povos de origem latina, de personalidade mais emocional, contrastando com povos de origem anglo-saxônica, de personalidade mais fria, racional. Outro aspecto interessante de se observar na Tabela 1 está no fato dos médicos, via de regra, estimarem valores mais elevados do que os enfermeiros, que por sua vez estimam valores mais elevados do que os psicólogos até o quadro clínico Úlcera peptídica. Neste escalonamento verificamos que a partir da região deste quadro clínico, os psicólogos tendem a superestimar os quadros clínicos em relação aos enfermeiros, e nos casos de quadros clínicos próximos da fase terminal, os psicólogos tendem a dar valores semelhantes aos dos médicos, chegando a ultrapassá-los em alguns casos como o câncer $(389,09 / 373,47)$ e AIDS (572,51/337,77). Levando-se em conta que as instruções são bastante claras para o fato de que a experiência pessoal deve ser levada em conta para julgar as doenças, verificamos que os médicos, pela própria característica profissional, tem grande contato com essas patologias e sua função é, se possível, eliminá-las ou então controlálas. Os enfermeiros, também tem contato com as doenças, porém sua responsabilidade profissional é um pouco menor, daí, provavelmente darem valores menores. Os psicólogos julgaram, em geral, mais baseados em suposições, pois não é uma constante o seu convívio com pacientes que possuam os quadros clínicos listados no instrumento. Provavelmente esta variação ocorreu devido a pouca importância dada à quadros clínicos como caspa e acne, não representando maiores conseqüências e por outro lado quadros clínicos como câncer e AIDS que gerariam angústias de alto cunho afetivo associadas à perdas e possíveis lutos.

Quanto a quantidade de respostas em branco deixadas no instrumento. Somente dois itens, carbúnculo e desvio sexual, não foram respondidos por quatro médicos. O primeiro item, talvez por desconhecimento deste quadro clínico, visto que atualmente é raro e o segundo provavelmente porque desvio sexual não é mais considerado doença. Dez enfermeiros deixaram de responder o item carbúnculo além de mononucleose, provavelmente devido ao desconhecimento destes diagnósticos e sete participantes não responderam ao item febre do feno, provavelmente pelo mesmo motivo. Quanto aos psicólogos, quatro deixaram de responder sobre psoríase, veia varicosa, mononucleose e febre do feno, enquanto que cinco participantes não responderam sobre carbúnculo e uremia, provavelmente devido ao seu desconhecimento desses quadros clínicos.

$\mathrm{Na}$ Tabela 2 estão apresentados os coeficientes de correlações de Pearson (r), calculados entre as médias geométricas obtidas das estimativas de magnitudes dos enfermeiros brasileiros (EM1), psicólogos brasileiros (EM2), médicos brasileiros (EM3), média geral brasileira
(EMB), médicos americanos (EMM), leigos americanos (EML) e média geral americana (EMA) variaram de 0,80 a 0,99 (as amostras americanas referem-se às utilizadas no estudo de WYLER et al. (1968). Nas Figuras 1, 2 e 3 estão apresentadas as funções de potência relacionando as estimativas de magnitudes das gravidades dos diferentes quadros clínicos efetuadas pela amostra brasileira com aquelas efetuadas pela amostra americana geral, amostra de médicos americanos e leigos americanos. Os três expoentes foram 1,22. Este valor indica portanto, que a amostra brasileira superestimou os quadros clínicos em relação as amostras americanas, ou seja, talvez a percepção de gravidades de quadros clínicos, leve-os culturalmente a situações mais estressantes ao lidar com elas.

Tabela 2 - Matriz das correlações de Pearson resultantes entre as médias geométricas obtidas das estimativas de magnitudes dos enfermeiros brasileiros (EM1), psicólogos brasileiros (EM2), médicos brasileiros (EM3), média geral brasileira (EMB), médicos americanos (EMM), leigos americanos (EML) e média geral americana (EMA)

\begin{tabular}{l|c|c|c|c|c|c|c}
\hline & EM1 & EM2 & EM3 & EMB & EMM & EML & EMA \\
\hline EM1 & - & 0,94 & 0,92 & 0,97 & 0,80 & 0,85 & 0,83 \\
EM2 & 0,94 & - & 0,93 & 0,98 & 0,84 & 0,88 & 0,87 \\
EM3 & 0,92 & 0,93 & - & 0,97 & 0,88 & 0,88 & 0,89 \\
EMB & 0,97 & 0,98 & 0,97 & - & 0,86 & 0,89 & 0,89 \\
EMM & 0,80 & 0,84 & 0,88 & 0,86 & - & 0,95 & 0,99 \\
EML & 0,85 & 0,88 & 0,88 & 0,89 & 0,89 & - & 0,99 \\
EMA & 0,83 & 0,87 & 0,89 & 0,89 & 0,99 & 0,99 & - \\
\hline
\end{tabular}

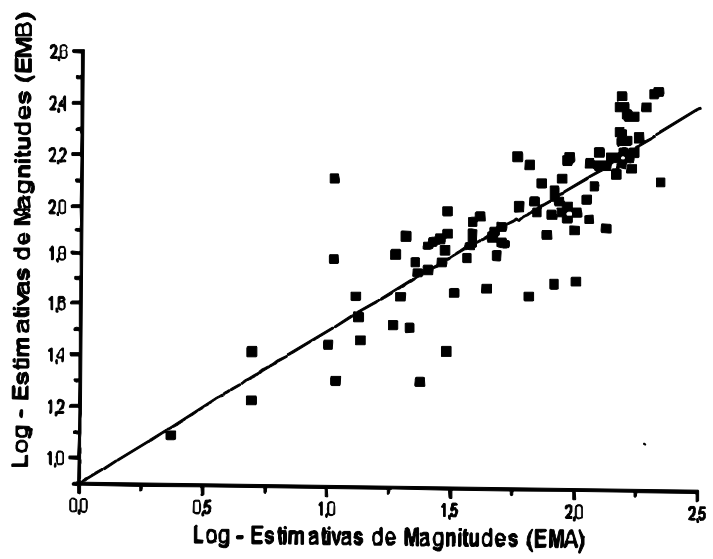

Figura 1 - Relação entre os logarítmos das médias geométricas gerais brasileiras (EMB) e os logarítmos das médias geométricas gerais americanas (EMA) 


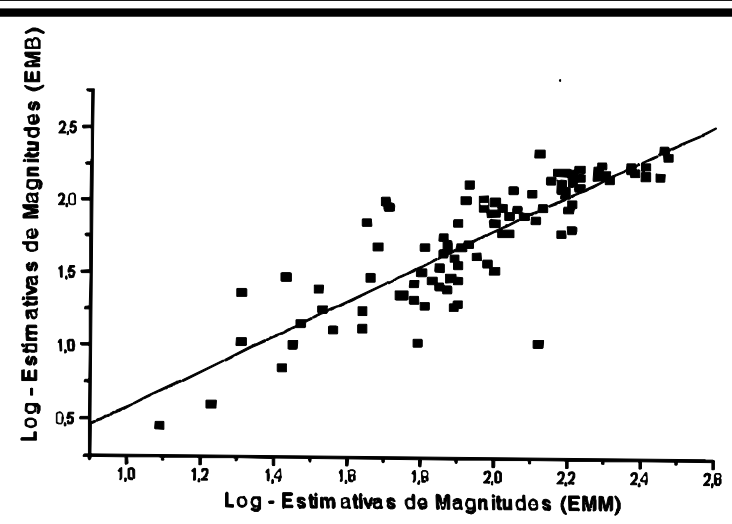

Figura 2 - Relação entre os logarítmos das médias geométricas gerais brasileiras (EMB) e os logarítmos das médias geométricas das estimativas de magnitudes dos médicos americanos (EMM)

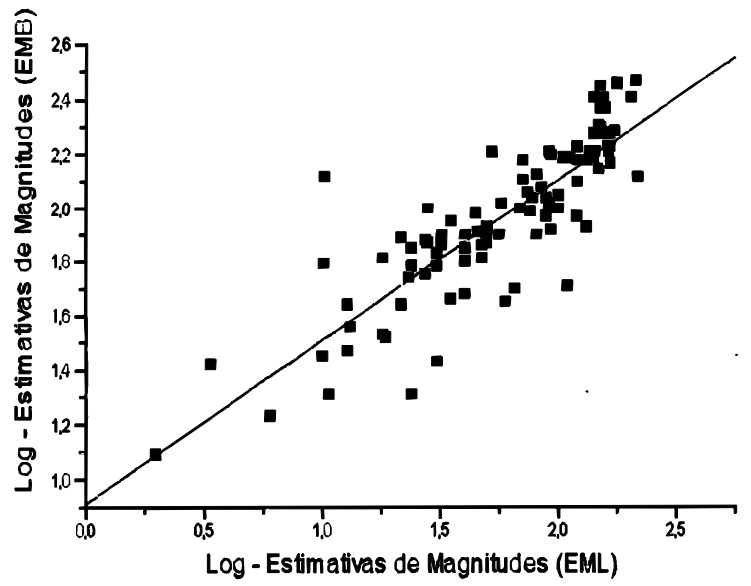

Figura 3 - Relação entre os logarítmos das médias geométricas gerais brasileiras (EMB) e os logarítmos das médias geométricas das estimativas de magnitudes dos leigos americanos (EML)

\section{ESTABILIDADE INTERCULTURAL}

\section{TEMPORAL}

$\mathbf{E}$

Na Tabela 3 apresentamos as comparações entre as três amostras, sendo a EMI amostra composta por estudantes de Psicologia de RIPPERE (1976), representantes dos ingleses; EMI amostra composta de médicos americanos de WYLER et al.(1968) e EMB a média geral brasileira (1996). Estão apresentados 12 quadros clínicos selecionados por Rippere (1976). A autora utilizou 15 quadros clínicos, tais como braço quebrado, perna quebrada, câncer no pulmão para facilitar a tarefa dos participantes, visto que eram do segundo ano de faculdade, tanto de Medicina como de Psicologia. O quadro clínico bronquite foi considerado o de menor gravidade, enquanto que, os quadros clínicos câncer e leucemia foram os de maior gravidade para as três amostras interculturais. Houve uma notada diferença, quanto às ordenações nos quadros clínicos pneumonia, diabetes e sífilis na amostra americana em relação às amostras inglesa e brasileira, porém não foi significativa, pois o coeficiente de concordância de Kendall (W) mostrou que, as diferentes amostras foram concordantes quanto à ordenação desses diferentes quadros clínicos (diagnósticos). Esse valor do coeficiente de concordância foi $\mathrm{W}=0,87(\mathrm{p}<0,001)$. Estes dados nos permitem testemunhar a estabilidade temporal e intercultural da escala de razão de severidade de quadros clínicos, pois os dados de WYLER et al. datam de 1968, enquanto que os dados de RIPPERE são de 1976, enquanto que os nossos dados são referentes a 1996, ou seja, a consistência dessa escala nos mostra uma grande estabilidade num período de 28 anos, além de ignorar fronteiras físicas e culturais, visto que os dados foram coletados nos Estados Unidos, Inglaterra e Brasil. Outra possível conclusão está no fato que, apesar dos avanços da medicina durante esse período, o conceito de graus de severidade de quadros clínicos permaneceu praticamente inalterado. Nas Figuras 4 e 5 estão apresentadas as funções de potência relacionando as estimativas de magnitudes das gravidades dos diferentes quadros clínicos efetuadas pela amostra inglesa (EMI) com aquelas efetuadas pela amostra americana de médicos (EMM), e a média geral brasileira (EMB). Os dois expoentes foram 1,15 e 1,08 respectivamente. Este valor indica portanto, que houve uma pequena superestimação na amostra brasileira em relação a amostra inglesa, novamente indicativo de questões culturais de cunho emocional, próprio dos latinos e há praticamente concordância entre as amostras inglesa e americana mostrando que, talvez, devido a mesma gênese cultural, as duas amostras se equivalem.

Tabela 3 - Média geométrica das estimativas de magnitudes das amostras inglesas (EMI), americanas (EMM) e brasileiras (EMB) e respectivas ordenações de posições (OP) das gravidades julgadas de cada quadro clínico no experimento de Rippere (1976)

\begin{tabular}{|c|c|c|c|c|c|c|}
\hline Diagnósticos & EMI & $\mathbf{O P}$ & EMM & $\mathbf{O P}$ & EMIB & $\mathbf{O P}$ \\
\hline Bronquite & 74,40 & $1^{\circ}$ & 37,20 & $1^{*}$ & 98,30 & $2^{*}$ \\
\hline Luxação & 83,20 & $2^{\circ}$ & 92,20 & $6^{\circ}$ & 51,39 & $1^{*}$ \\
\hline Úkera & $100, \infty$ & $3^{*}$ & 100,00 & $7^{\circ}$ & 100,00 & $3^{8}$ \\
\hline Hïertenxĩo & 102,80 & $4^{*}$ & 91,20 & $5^{\circ}$ & 104,92 & $5^{8}$ \\
\hline Pneumoria & 110,60 & $5^{8}$ & 59,60 & $2^{\circ}$ & 104,31 & $4^{\circ}$ \\
\hline Sifilis & 111,20 & $6^{8}$ & 74,40 & $4^{\circ}$ & 128,51 & $6^{\circ}$ \\
\hline Tuberculose & 120,00 & $7^{\circ}$ & 122,60 & $8^{\circ}$ & 150,71 & $7^{\circ}$ \\
\hline Diabetes & 124,20 & $8^{\circ}$ & 59,60 & $3^{\circ}$ & 152,52 & $8^{\circ}$ \\
\hline Derrame & 180,00 & $9^{\circ}$ & 153,20 & $9^{\circ}$ & 198,43 & $10^{\circ}$ \\
\hline Cósulo nos vasos & 191,60 & $10^{\circ}$ & 164,00 & $10^{\circ}$ & 155,78 & $9^{\circ}$ \\
\hline Cânoer & 230,80 & $11^{\circ}$ & 210,00 & $11^{\circ}$ & 292,02 & $12^{\circ}$ \\
\hline Leucenia & 233,20 & $12^{\circ}$ & 232,00 & $12^{\circ}$ & 289,04 & $11^{*}$ \\
\hline
\end{tabular}

Obs: os dados de WYLER et al. (1968), além dos dados de RIPPERE (1976) foram divididos por 5, pois foi adotado o módulo 500 para Úlcera, enquanto que em nossa pesquisa foi 100 


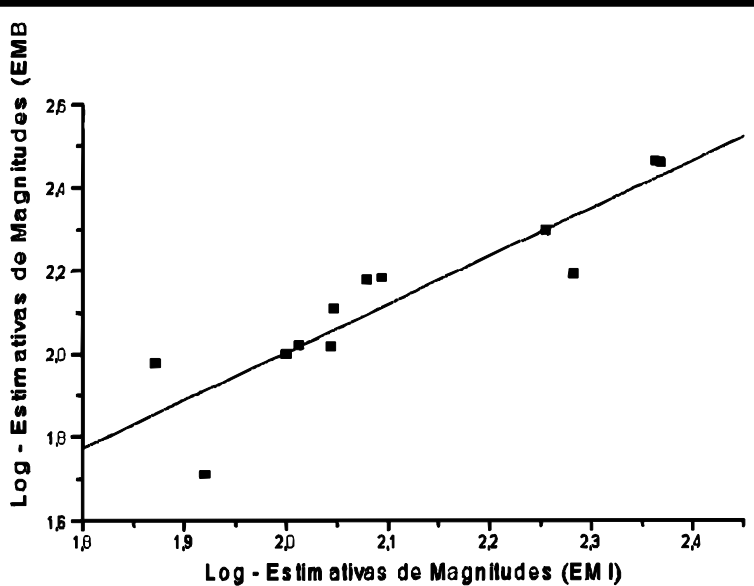

Figura 4 - Relação entre os logarítmos das médias geométricas gerais brasileiras (EMB) e os logarítmos das médias geométricas das estimativas de magnitudes da amostra de Rippere (EMI)

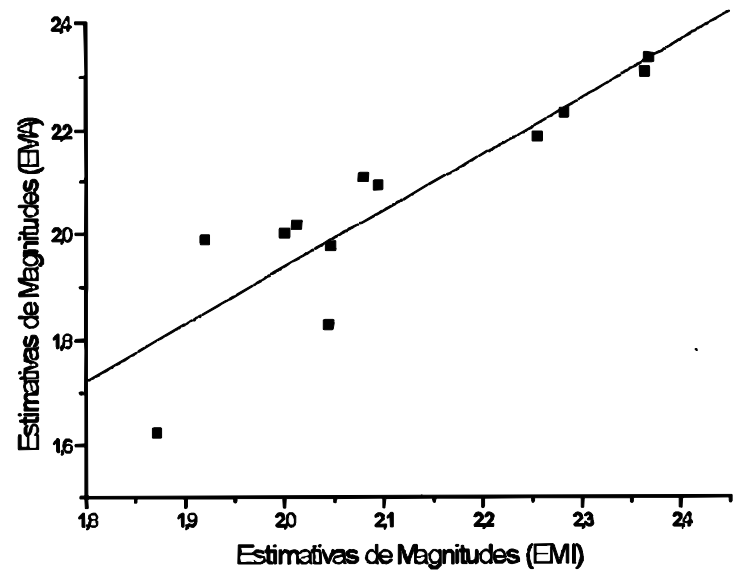

Figura 5 - Relação entre os logarítmos das médias geométricas gerais americanas (EMA) e os logarítmos das médias geométricas das estimativas de magnitudes da amostra de Rippere (EMI)

\section{MAGNITUDE ESTIMATION OF THE SEVERITY OF CLINICAL ILLNESS: A PSYCHOPHYSICAL APPROACH}

The severity of clinical illness was evaluated through the psychophysics method of magnitude estimation. The purpose of this experiment was: 1) to compare the scales derived from right judgement (magnitude estimation) used in three different Brazilian samples; 2) to verify the stability and agreement of severity estimations of clinical situations judged in Brazil, USA and England. In order to develop this study, professionals who work in the areas of psychology, nursing and medicine were invited. The lower coefficient of Pearson's correlation among the groups were of 0,88 the and the greater of 0,94. The exponents found between the Brazilian and American samples were of 1,22 and between the Brazilian and English samples of 1,15.

KEY WORDS: severity of diseases, magnitude estimation, psychophysics

\section{ESTIMACIÓN DE LA MAGNITUD DE LA GRAVEDAD DE CUADROS CLÍNICOS: UN ENFOQUE DE LA PSICOFÍSICA CLÍNICA}

En este experimento la gravedad de los cuadros clínicos fue evaluada a través del método psicofísico de estimación de magnitudes. Los objetivos de este experimento fueron: 1) comparar escalas derivadas de juzgamientos de razón (estimativas de magnitudes), hechos por tres diferentes muestras brasileñas; 2) verificar la estabilidad y la concordancia de las estimativas de la gravedad de cuadros clínicos juzgados en Brasil, EUA e Inglaterra. Así, fueron invitados profesionales actúan en las áreas de psicología, enfermería y medicina. El coeficiente de correlación de Pearson entre los grupos fue de 0,88 a menor y 0,94 a mayor. Los exponentes encontrados entre las muestras brasileñas y americanas fue de 1,22 y entre las muestras brasileñas e inglesas fue de 1,15 .

TÉRMINOS CLAVES: gravedad de la enfermedad, estimación de magnitud, psicofísica

\section{REFERÊNCIAS BIBLIOGRÁFICAS}

01. SOUSA, F.A.E.F. Prestígio profissional do enfermeiro: um enfoque da psicofísica social. Ribeirão Preto, 1993. 197 p. Tese (Doutourado) - Escola de Enfermagem de Ribeirão Preto, Universidade de São Paulo.

02. SOUSA, F.A.E.F.; SILVA, J.A.da .Uso e aplicação da metodologia psicofísica na pesquisa em Enfermagem. Rev.latino-am.enfermagem, v. 4, p.147-178, 1996.
03. RIPPERE, V. Scaling the seriousness of illness: a methodological study. Journal of Psychossomatic Research, v. 9, p. 567-573, 1976.

04. STEVENS, S.S. A metric for the social consensus. Science, v. 151, p. 530-541, 1966. In: STEVENS, S.S., G (ed). Psychophysics: introduction to its perceptual, neural and social prospects. New York: Wiley, 1975. 
05. VOLICER, B.J.; BOHANNON, M.W. A hospital stress rating scale. Nursing Research, v. 24, p. 352-364, 1975.

06. WYLER, A.R.; MASUDA, M.; HOLMES, T.H. Seriousness of illness rating scale. Journal of Psychossomatic Research, v. 11, p. 363-374, 1968.
07. WYLER, A.R.; MASUDA, M.; HOLMES, T.H. Seriousness of illness rating scale: reproducibility. Journal of Psychossomatic Research, v. 14, p. 59-64, 1970.

08. WYLER, A.R.; MASUDA, M.; HOLMES, T.H. Magnitude of life events and seriousness of illness. Psychossomatic Medicine, v. 33, p.115122, 1971. 